\title{
On the onset of incipient fluidization
}

\begin{abstract}
The onset of incipient fluidization is investigated theoretically and simulated by a computational fluid dynamics (CFD) procedure. The onset of incipient instability in a particle bed is preceded by stable gas diffusion in the interstices and is caused by a critical momentum force that may overcome the inertia of the particles. The critical momentum force is provided by the critical superficial gas velocity Uc in the form of critical mass flux of diffusion. It is found that the first movement of particles may be predicted by a critical transient Rayleigh number determined by a critical superficial velocity equals to the minimum fluidization velocity, Umf. The onset of incipient fluidization was found to occur at a critical transient Rayleigh number of 3.1, which is close to the lowest theoretical value for buoyancy convection in a porous medium bounded by free surfaces. Consequently the onset times of incipient fluidization may be predicted accurately. The finding has been found to be supported by the present CFD study, past experiments and simulations in the literature.
\end{abstract}

Keyword: Onset; Incipient fluidization; Transient Rayleigh number 